\title{
Ext A LA YONEDA WITHOUT THE SCHANUEL LEMMA
}

\author{
RUDOLF FRITSCH
}

\begin{abstract}
By means of a more precise treatment of the congruence relation between $n$-extensions we show that it is possible to avoid the Schanuel Lemma for proving some results on the functors Ext.
\end{abstract}

An $n$-extension $\mathbf{E}$ from $D$ to $C$ is an exact sequence of the form

$$
0 \rightarrow C \rightarrow E_{1} \rightarrow E_{2} \rightarrow \cdots \rightarrow E_{n} \rightarrow D \rightarrow 0
$$

in an abelian category. The idea to use congruence classes $n$-extensions as elements of $\operatorname{Ext}^{n}(D, C)$ is due to Yoneda ([7], [8]; cf. also Buchsbaum [4] and Mac Lane [5, Chapter III]). ${ }^{1}$ In studying Ext $^{n}$ from this point of view, the Schanuel Lemma (Mitchell [6, Chapter VII, Lemma 4.1]) plays an essential role: firstly, in order to get the exactness of the long sequence (Mitchell 1.c. $\S 5$ ), secondly, for proving that a congruence between two $n$-extensions can be realized in two steps (Brinkmann [2]). However the Schanuel Lemma and its proof are of a highly technical nature. Therefore, in the following we propose a more straightforward way to these results, the key to which lies in the following

TheOREM. Two n-extensions $\mathbf{E}^{1}$ and $\mathbf{E}^{2}$ are congruent over an n-extension if and only if they are congruent under an n-extension.

Here "congruence of $\mathbf{E}^{1}$ and $\mathbf{E}^{2}$ over (under) an $n$-extension" means the existence of an $n$-extension $\mathbf{E}$ and of morphisms $\mathbf{f}^{i}(i=1,2)$ with fixed ends (in the sense of Mitchell 1.c. \$3) forming the diagram

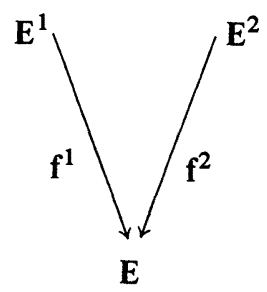

resp.

Received by the editors October 7, 1974 and, in revised form, November 21, 1974.

AMS (MOS) subject classifications (1970). Primary 18G15.

Key words and phrases. $n$-extension, congruence, morphism with fixed ends.

${ }^{1}$ In case $n=1$ this goes back to Baer [1]. A very nice way to get the group structure on $\operatorname{Ext}^{n}(D, C)$ is described by Brinkmann in [3].

(c) American Mathematical Society 1976 


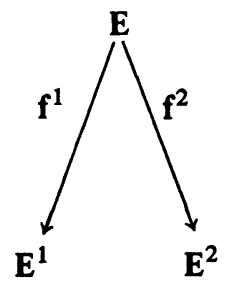

An immediate consequence of this theorem is that "congruence over" and "congruence under" are equivalence relations and that they coincide. This is the precise statement of the result on the realizability of congruences quoted above. Moreover, the exactness of the long sequence can be easily deduced from this theorem.

We demonstrate the step of its proof for which Mitchell [6, p. 179] uses the Schanuel Lemma.

Let $B$ be the short exact sequence

$$
0 \rightarrow A \stackrel{i}{\rightarrow} B \stackrel{p}{\rightarrow} C \rightarrow 0
$$

and let $\mathbf{E}$ be an $n$-extension of the form (1). Let further the spliced $(n+1)$-extension $\mathbf{B E}$ represent the zero of $\operatorname{Ext}^{n+1}(D, A)$. Then, we have to show that $\mathbf{E}$ is congruent to $p \mathbf{E}^{\prime}$ for some $n$-extension $\mathbf{E}^{\prime}$ from $D$ to $B$. By means of the theorem, we have an $(n+1)$-extension $\hat{\mathbf{E}}$ from $D$ to $A$ such that $B E$ and $O$ (the latter having the form

(4)

$$
0 \rightarrow A \rightarrow A \rightarrow 0 \rightarrow \cdots \rightarrow 0 \rightarrow D \rightarrow D \rightarrow 0)
$$

are congruent under $\hat{\mathbf{E}}$.

The existence of a morphism from $\hat{\mathbf{E}}$ to the extension $\mathbf{0}$ implies that $\hat{\mathbf{E}}$ can be decomposed as

$$
\hat{\mathbf{E}}=\overline{\mathbf{B}} \overline{\mathbf{E}}
$$

where $\bar{B}$ is of the form

$$
0 \rightarrow A \underset{i_{A}}{\rightarrow} A \oplus \overline{C_{\vec{p}}} \underset{\bar{C}}{\rightarrow} \bar{C} \rightarrow 0 .
$$

Now, the morphism from $\hat{\mathbf{E}}$ to $\mathbf{B E}$ with fixed ends induces a map $f: \bar{C} \rightarrow C$ which factors through $p$, i.e. we have

$$
f=p g
$$

for some map $g: \bar{C} \rightarrow B$. Then,

$$
\mathbf{E}^{\prime}=g \overline{\mathbf{E}}
$$

is an $n$-extension of the desired kind.

PROOF OF THE THEOREM. We prove that "congruence over" implies "congruence under"; the converse is dual. Thus, let a diagram of the form (2o) be given. We may assume that the $f^{i}$ consist of isomorphisms except at only two places (Mitchell 1.c. Proposition 3.1), $k^{i}$ and $k^{i}+1\left(i=1,2 ; 1 \leqslant k^{i}\right.$ $<n$ ) say. We may assume

$$
k^{1} \leqslant k^{2} \leqslant k^{1}+1
$$

In case

$$
k^{2}=k^{1}+1
$$

we split all the $n$-extensions involved into a left part of length $k^{2}$ and the remaining right part. We denote the left and right parts by the subscripts 
and, respectively. Then there is a morphism $h$, such that

$$
\mathbf{E}_{l}^{2}=\mathbf{E}_{l} h
$$

and

$$
h \mathbf{E}_{r}^{2}=\mathbf{E}_{r} .
$$

This shows that the $\mathbf{E}^{i}(i=1,2)$ are congruent under $\left(E_{l}^{1} h\right) E_{r}^{2}$. If

$$
k^{2}=k^{1}
$$

we can restrict our attention to the case $n=2$. There, we have the following diagram:

$$
\begin{array}{ccccccccc}
0 \rightarrow & C & \rightarrow & E_{1}^{1} & \overrightarrow{g^{1}} & E_{2}^{1} & \overrightarrow{p^{1}} & D & \rightarrow 0 \\
& \| & & \downarrow f_{1}^{1} & & \downarrow f_{2}^{1} & & \| & \\
0 \rightarrow & C & \rightarrow & E_{1} & \rightarrow g & E_{2} & \vec{p} & D & \rightarrow 0 \\
& \| & & \uparrow f_{1}^{2} & & \uparrow f_{2}^{2} & & \| & \\
0 \rightarrow & C & \overrightarrow{i^{2}} & E_{1}^{2} & \overrightarrow{g^{2}} & E_{2}^{2} & \overrightarrow{p^{2}} & D & \rightarrow 0
\end{array}
$$

In this situation we form the pullback

$$
\begin{array}{lll}
E_{2}^{\prime} & \stackrel{h}{\rightarrow} & E_{2}^{1} \oplus E_{2}^{2} \\
\downarrow & & \downarrow\left\langle f_{2}^{1},-f_{2}^{2}\right\rangle \\
E_{1} & \rightarrow & E_{2}
\end{array}
$$

Then, on the one hand, we have a map $g^{\prime}: E_{1}^{1} \oplus E_{1}^{2} \rightarrow E_{2}^{\prime}$, given by

$$
g^{\prime}=\left(\left\langle f_{1}^{1},-f_{1}^{2}\right\rangle, g^{1} \oplus g^{2}\right) ;
$$

on the other hand, the composition

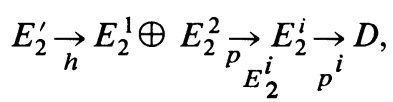

which is independent of $i(i=1,2)$, yields a map $p^{\prime}: E_{2}^{\prime} \rightarrow D$. This leads to a 2-extension

$$
0 \rightarrow C \underset{\left(i^{1}, i^{2}\right)}{\rightarrow} E_{1}^{1} \oplus \underset{g^{\prime}}{2} \underset{2}{\rightarrow} \underset{p^{\prime}}{\rightarrow} D \rightarrow 0
$$

under which the given 2-extensions $\mathbf{E}^{1}$ and $\mathbf{E}^{2}$ are congruent. The exactness of (18) can be verified directly in the case of modules by means of diagram chasing; then the translation into the general case of abelian categories is obvious but also tedious.

The author is indebted to H.-B. Brinkmann and S. Mac Lane for helpful conversations in preparing this note.

\section{REFERENCES}

1. R. Baer, Erweiterung von Gruppen und ihren Isomorphismen, Math. Z. 38 (1934), 375-416.

2. H.-B. Brinkmann, Equivalence of n-extensions, Arch. Math. (Basel) 19 (1968), 624-626 (1969). MR 39 \#2839. 
3. H.-B. Brinkmann, Baer addition of extensions, Manuscripta Math. 1 (1969), 99-109. MR 39 \#244.

$\rightarrow$ D. A. Buchsbaum, A note on homology in categories, Ann. of Math. (2) 69 (1959), 66-74. MR 25 \#3974.

5. S. Mac Lane, Homology, Die Grundlehren der math. Wissenschaften in Einzeldarstellungen, Band 114, Springer-Verlag, Berlin and New York, 1967.

6. B. Mitchell, Theory of categories, Pure and Appl. Math., vol. 17, Academic Press, New York and London, 1965. MR 34 \#2647.

7. N. Yoneda, On the homology theory of modules, J. Fac. Sci. Univ. Tokyo Sect. I 7 (1954), 193-227. MR 16, 947.

8. , On Ext and exact sequences, J. Fac. Sci. Univ. Tokyo Sect. I 8 (1960), 507-576. MR 37 \#1445.

FACHBEREICH MATEMATIK, UNIVERSIT ÄT KONSTANZ, POSTFACH 7733, D-7750 KONSTANZ, WEST GERMANY 